\title{
Identification of large intergenic non-coding RNAs in bovine muscle using next-generation transcriptomic sequencing
}

\author{
Coline Billerey ${ }^{1,2,3,4+}$, Mekki Boussaha ${ }^{1,2+}$, Diane Esquerré5, ${ }^{5,6}$, Emmanuelle Rebours ${ }^{1,2}$, Anis Djari ${ }^{7}$,
} Cédric Meersseman ${ }^{1,2}$, Christophe Klopp ${ }^{7}$, Daniel Gautheret ${ }^{3,4}$ and Dominique Rocha ${ }^{1,2^{*}}$

\begin{abstract}
Background: The advent of large-scale gene expression technologies has helped to reveal in eukaryotic cells, the existence of thousands of non-coding transcripts, whose function and significance remain mostly poorly understood. Among these non-coding transcripts, long non-coding RNAs (IncRNAs) are the least well-studied but are emerging as key regulators of diverse cellular processes. In the present study, we performed a survey in bovine Longissimus thoraci of lincRNAs (long intergenic non-coding RNAs not overlapping protein-coding transcripts). To our knowledge, this represents the first such study in bovine muscle.

Results: To identify lincRNAs, we used paired-end RNA sequencing (RNA-Seq) to explore the transcriptomes of Longissimus thoraci from nine Limousin bull calves. Approximately 14-45 million paired-end reads were obtained per library. A total of 30,548 different transcripts were identified. Using a computational pipeline, we defined a stringent set of 584 different lincRNAs with 418 lincRNAs found in all nine muscle samples. Bovine lincRNAs share characteristics seen in their mammalian counterparts: relatively short transcript and gene lengths, low exon number and significantly lower expression, compared to protein-encoding genes. As for the first time, our study identified lincRNAs from nine different samples from the same tissue, it is possible to analyse the inter-individual variability of the gene expression level of the identified lincRNAs. Interestingly, there was a significant difference when we compared the expression variation of the 418 lincRNAs with the 10,775 known selected protein-encoding genes found in all muscle samples. In addition, we found 2,083 pairs of lincRNA/proteinencoding genes showing a highly significant correlated expression. Fourteen lincRNAs were selected and 13 were validated by RT-PCR. Some of the lincRNAs expressed in muscle are located within quantitative trait loci for meat quality traits.

Conclusions: Our study provides a glimpse into the lincRNA content of bovine muscle and will facilitate future experimental studies to unravel the function of these molecules. It may prove useful to elucidate their effect on mechanisms underlying the genetic variability of meat quality traits. This catalog will complement the list of lincRNAs already discovered in cattle and therefore will help to better annotate the bovine genome.
\end{abstract}

Keywords: Cattle, Muscle, RNA-Seq, Beef, Long non-coding RNA

\footnotetext{
* Correspondence: dominique.rocha@jouy.inra.fr

${ }^{\dagger}$ Equal contributors

'INRA, UMR1313, Unité Génétique Animale et Biologie Intégrative, Domaine de Vilvert, F-78352 Jouy-en-Josas, France

${ }^{2}$ AgroParisTech, UMR1313, Unité Génétique Animale et Biologie Intégrative,

Domaine de Vilvert, F-78352 Jouy-en-Josas, France

Full list of author information is available at the end of the article
} 


\section{Background}

Over the past decade, genome-wide transcriptional studies discovered that a large fraction of the eukaryotic genomes is transcribed in a heterogeneous population of noncoding RNAs (ncRNAs). These are transcripts that are not translated into a protein but act as functional RNAs. ncRNAs shorter than 200 nucleotides are usually identified as small/short ncRNA and include PIWIinteracting RNAs (piRNAs), endogeneous small interfering RNAs (siRNAs) and microRNAs (miRNAs) but also classical ncRNAs, such as ribosomal RNAs (rRNAs), transfer RNAs (tRNAs) and small nucleolar RNAs (snoRNAs); whereas those longer than 200 nucleotides are classified as long ncRNAs (lncRNAs). LncRNAs can be classified as lincRNAs (long intergenic non-coding RNAs) that are transcribed adjacent to protein-coding genes, eRNAs (enhancer RNAs that are transcribed within the enhancer regions), intronic lncRNAs (transcribed within the introns of protein-coding genes) and antisense lncRNAs (transcribed from the opposite genomic strand relative to proteincoding genes) [1,2]. In the past few years, an increasing number of lncRNAs have been discovered in eukaryotic organisms, ranging from nematodes to humans [3-17]. For example, the most recent report of the ENCODE (Encyclopedia of DNA Elements) project published in September 2012, described 9,640 lncRNA loci in comparison to 20,687 protein-coding genes in 15 human cell lines [14-16].

LncRNAs can be polyadenylated or non-polyadenylated $[17,18]$, spliced or mono-exonic unspliced and the expression level of individual lncRNAs is generally lower than the level of expression of the typical protein-coding mRNAs [17,19-22], and some lncRNAs have high tissue specificity [21-24].

Despite the fact that only few IncRNAs have been characterized experimentally in detail to date, it is already known that they can act via diverse mechanisms [25] and can play regulatory and structural roles in almost every important biological process, such as Xchromosome inactivation and genomic imprinting, nuclear compartmentalization and architecture, cell fate specification, RNA splicing, translational control, and chromatin modification [26].

Because of the key role of IncRNAs in regulation of gene expression and therefore possible impact on phenotypes, it is important to identify most lncRNAs. Catalogues of IncRNA have been established for many species, including cattle [27-29]. For example, Huang et al. (2012) have identified a total of 449 putative lncRNAs located in 405 intergenic regions using public bovinespecific expressed sequence tags sequences [28]. More recently, Weikard et al. (2013) predicted more than 4,000 potential lncRNAs in bovine skin using RNA-Seq data [29]. The current number of bovine lncRNAs identified is rather low compared to more than 9,000 lncRNAs found in human, suggesting that more efforts are needed to discover all bovine lncRNAs.

In the present study, we identified lncRNAs in bovine Longissimus thoraci, using a whole-transcriptome sequencing approach. To our knowledge, this represents the first study done in bovine muscle. For this purpose, muscle samples from nine different Limousin bulls were analysed. We have identified more than 500 different lincRNAs and 13 out of the 14 selected lincRNAs were validated experimentally. The RNA-Seq data and the collection of newly discovered lincRNAs improve the genomic resources available for cattle, especially for beef breeds. This collection of lincRNAs may prove useful to study their link with genetic variability of meat quality traits.

\section{Results and discussion}

RNA sequencing and assembly of a muscle transcriptome To identify lincRNAs expressed in the bovine Longissimus thoraci, we used paired-end RNA sequencing (RNA-Seq) from nine Limousin bull calves. We used already published data from three Limousin animals [30] and poly(A)enriched mRNA from six new Limousin bull calves were retrotranscribed and subjected to high-throughput sequencing. The six RNA-Seq libraries were barcode-tagged and sequenced on two lanes (3 libraries per lane) of an Illumina HiSeq2000 sequencer. The reads were then de-multiplexed to assign reads to each sequenced sample according to its barcode index.

Sequencing of all nine cDNA libraries generated a total of 300,941,530 raw paired-end reads with a length of 100 bases, resulting in a total of 60 gigabases. Approximately 14 to 45 million paired-end reads were obtained for each library. The reads were then aligned using TopHat [31] onto the bovine UMD3.1 reference genome sequence. $65 \%$ to $75 \%$ of the reads were aligned onto the bovine genome, and $82 \%$ to $92 \%$ of the mapped reads were aligned properly paired (Table 1 ). Transcripts were reconstructed using Cufflinks [32], resulting in the assembly of a total number of 131,753 transcripts (30,548 different genes) with at least one paired-end read.

Similar RNA-Seq read mapping rates were obtained in other RNA-Seq bovine studies [33-38]. For example, Baldwin and collaborators found by sequencing the rumen epithelium that $\sim 71 \%$ of the reads uniquely mapped to specific regions of the bovine genome [36]. Interestingly a comparable number of genes has been detected in bovine skin in a RNA-Seq project using a similar sequencing coverage and bioinformatics pipeline [29].

Raw gene expression levels were estimated by measuring the normalised count number for each transcript (number of reads per transcript divided by the total number of mapped reads, for each sample). The five 
Table 1 Summary of reads mapping to the bovine transcriptomes

\begin{tabular}{lccccccccc}
\hline & LIM1 & LIM2 & LIM3 & LIM4 & LIM5 & LIM6 & LIM7 & LIM8 & LIM9 \\
\hline Number of reads & $86,352,760$ & $72,251,962$ & $90,678,870$ & $74,649,210$ & $72,416,218$ & $80,220,062$ & $38,198,732$ & $27,278,276$ & $59,836,970$ \\
Number of bases (in Gb) & 8.64 & 7.23 & 9.07 & 7.46 & 7.24 & 8.02 & 3.82 & 2.73 & 5.98 \\
Number of mapped reads & $65,739,933$ & $54,576,643$ & $68,916,620$ & $61,346,058$ & $50,206,044$ & $61,543,271$ & $30,174,318$ & $21,840,568$ & $46,205,282$ \\
\% mapped reads & 76.13 & 75.54 & 76 & 82.18 & 69.33 & 76.72 & 78.99 & 80.07 & 77.22 \\
Number of uniquely mapped reads & $61,587,716$ & $51,574,445$ & $65,139,874$ & $56,117,512$ & $47,169,853$ & $58,093,383$ & $28,421,388$ & $20,558,269$ & $43,701,020$ \\
\% uniquely mapped reads & 70.82 & 71.38 & 71.84 & 75.17 & 65.14 & 72.42 & 74.4 & 75.36 & 73.03 \\
Number of uniquely mapped & $32,792,300$ & $26,484,662$ & $32,399,058$ & $25,646,394$ & $24,089,814$ & $30,477,552$ & $15,891,664$ & $12,590,540$ & $23,843,178$ \\
paired-reads & & & & & & & & & \\
\% uniquely mapped paired-reads & 86.22 & 84.15 & 82.29 & 90.86 & 85.68 & 87.09 & 82.47 & 92.04 & 86.62 \\
\hline
\end{tabular}

most frequent transcripts are shown in Table 2. These five genes (actin alpha skeletal muscle, myosin 1 and myosin 2, nebulin, titin) represent nearly $20 \%$ of all sequencing reads mapped to the bovine genome and are all associated with muscle structure. These results were consistent with the physiological role of genes expected in the surveyed tissue.

Not all genes were expressed among the nine selected samples. Transcripts corresponding to 20,907 different genes were detected in all nine samples, while approximately $32 \%$ of the genes were expressed in only some of the samples, including 1,443 different genes $(\sim 5 \%)$ only expressed in one sample.

Transcript models predicted with Cufflinks for each sample were sorted into different categories using the bovine genome annotation (Table 3 ). Approximately between $57-66 \%$ (mean +/- SD: $61 \%+/-3 \%$ ) of the transcripts correspond to already known transcripts. Surprisingly, between $\sim 18-22 \%$ (mean +/- SD: $20 \%+/-1 \%$ ) of the transcript models generated correspond to novel isoforms of known genes presumably from alternative splicing events (“j” class). This indicates that the bovine genome remains poorly annotated and that a large number of new transcript isoforms are still to be described. Interestingly, more than $2-4 \%$ (mean +/- SD: $3 \%+/-1 \%$ ) of the transcript models are predicted as unknown intergenic transcripts ("u" class). It is notable that some transcripts are classified into different categories. For example, a transcript model could be part of an already known transcript and at the same time it could be part of the novel isoform category, as the RNA-Seq data indicates a novel exon.

\section{Identification of putative lincRNAs}

To identify lncRNAs, we developed a stringent filtering pipeline to discard transcripts with evidence for proteincoding potential. We identify putative lncRNAs by considering their open reading frame, their phylogenetic conservation across species and homology with known proteins and protein domains. The reads were generated from non-directional RNA-Seq libraries, we therefore focus our effort on unknown intergenic transcripts and therefore could only identify putative lincRNAs.

First, a minimal transcript size criterion was applied. Transcripts with multiple exons and larger than $200 \mathrm{nt}$ were used. This analysis resulted in the identification of 2,291 putative multi-exonic intergenic transcripts $(1,127$ different loci).

Second, we used PhyloCSF to score the coding potential of unknown multi-exonic transcripts using multispecies alignments. PhyloCSF scores were calculated for the 2,291 putative multi-exonic intergenic transcripts and two control sets, one of 10,000 known proteincoding genes found in our RNA-Seq libraries and one with 438 already known bovine lncRNAs [28]. We set the PhyloCSF threshold empirically to a value retaining $73 \%$ of the known bovine ncRNAs while removing $7 \%$ of protein-coding transcripts. This filter retained 1,383 putative non-coding transcripts (798 different loci).

Third, we used CPAT on the same 2,291 putative multi-exonic intergenic transcripts, in order to assess their coding potential with a second prediction method. To determine the optimum cut-off value, CPAT was trained using a set of 10,000 bovine known protein-encoding

Table 2 Top five transcripts with most assigned reads

\begin{tabular}{lcccc}
\hline Locus & Gene name & Gene symbol & Number of reads (9 samples) & \% total number of reads \\
\hline XLOC_016519 & titin & TTN & $8,065,194$ & 6.07 \\
XLOC_015363 & myosin 1 & MYH1 & $5,127,160$ & 3.86 \\
XLOC_016610 & nebulin & NEB & $4,195,679$ & 3.16 \\
XLOC_015364 & myosin 2 & MYH2 & $3,787,472$ & 2.85 \\
XLOC_025531 & actin, alpha skeletal muscle & ACTA1 & $3,549,948$ & 2.67 \\
\hline
\end{tabular}


Table 3 Number of mapped sequencing reads for each different class of assembled transcripts

\begin{tabular}{|c|c|c|c|c|c|c|c|c|c|}
\hline & LIM1 & LIM2 & LIM3 & LIM4 & LIM5 & LIM6 & LIM7 & LIM8 & LIM9 \\
\hline \multicolumn{10}{|c|}{ Code } \\
\hline e & 841 & 898 & 884 & 711 & 904 & 757 & 655 & 547 & 741 \\
\hline$=$ & 26,052 & 26,029 & 26,072 & 26,075 & 26,237 & 26,064 & 2,6158 & 26,139 & 2,6117 \\
\hline$x$ & 285 & 276 & 250 & 227 & 278 & 271 & 195 & 177 & 247 \\
\hline 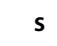 & 2 & 2 & 4 & 2 & 5 & 3 & 3 & 2 & 3 \\
\hline $\mathbf{j}$ & 9,262 & 9,660 & 9,476 & 9,008 & 7,811 & 9,121 & 8,111 & 8,223 & 8,753 \\
\hline c & 2 & 2 & 2 & 2 & 2 & 3 & 3 & 2 & 3 \\
\hline$p$ & 714 & 629 & 678 & 693 & 749 & 711 & 529 & 670 & 615 \\
\hline u & 1,459 & 1,510 & 1,988 & 1,123 & 1,842 & 1,462 & 868 & 1,058 & 985 \\
\hline . & 3,577 & 3,411 & 3,585 & 3,274 & 3,690 & 3,674 & 2,344 & 2,079 & 3,296 \\
\hline o & 413 & 434 & 448 & 377 & 354 & 427 & 317 & 288 & 362 \\
\hline $\mathbf{i}$ & 1,611 & 1,701 & 2,271 & 1,316 & 2,369 & 1,725 & 967 & 603 & 1,446 \\
\hline
\end{tabular}

e, single exon overlapping a reference exon and at least $10 \mathrm{bp}$ of a reference intron indicating a possible pre-mRNA fragment; $=$, complete match of intron chain; $\mathbf{x}$, exonic overlap with reference on the opposite strand; $\mathbf{s}$, an intron of the transfrag overlaps a reference intron on the opposite strand (likely due to read mapping errors); $\mathbf{j}$, potentially novel isoform; $\mathbf{c}$, contained in reference; $\mathbf{p}$, possible polymerase run-on fragment (within 2 kb of a reference transcript); $\mathbf{u}$, unknown intergenic transcript; ., tracking file only, indicates multiple classifications; $\mathbf{0}$, generic exonic overlap with a reference transcript; $\mathbf{i}$, a single exon transcript falling entirely within a reference intron.

transcripts and a set of 10,000 bovine non-coding sequences and a 10-fold cross-validation analysis was performed to estimate the prediction accuracy. A cut-off value of 0.348 was selected, maximising specificity and sensitivity (98.4\%) (Additional file 1: Figure S1). This procedure identified 2,085 transcripts (1,060 different loci) as potential non-coding RNAs. The intersection of PhyloCSF and CPAT predicted 1,330 transcripts (773 different loci) as potential non-coding RNAs.

Finally, we removed any remaining transcripts of uncertain coding potential that had similarity to known proteins or protein domains recorded in the Pfam database. The resulting set contained 1,277 transcript models corresponding to 584 different putative non-coding genes (Additional file 2: Table S1). There is a possibility that some real lincRNAs would have not been detected because of our stringent selection criteria. For example, some real lincRNAs could be lost owing to the chosen minimum transcript size. We might also have missed some lowly expressed lincRNA genes due to our moderate sequencing depth.

Comparison of the genomic position of the 584 different genes encoding putative lincRNAs found in bovine Longissimus thoraci with mapping positions of previously identified bovine lncRNAs publicly available in the NONCODE database (release 4) [39] show that $163(\sim 28 \%)$ of our lincRNAs overlap with previously described bovine non-coding RNA genes (Additional file 3: Table S2).

\section{Characterisation of identified lincRNAs}

The chromosomal location of the genes encoding these 584 putative lincRNAs is presented in the Additional file 4:
Figure S2. The chromosomal distribution usually reflects the gene content of the chromosomes: larger chromosomes have more lincRNA loci than shorter chromosomes. For example, we found 35 genes encoding putative lincRNAs on BTA10, whereas only 10 on BTAX.

Previous studies have shown that genes encoding lncRNAs are shorter in length, have shorter transcripts and have fewer exons than protein-coding genes $[6,11,40]$. To determine whether the bovine muscle lincRNAs we detected have the same features, we compared the size of 584 lincRNA genes to 15,358 protein-encoding genes detected in our RNA-Seq data. The lincRNAs represent much shorter gene length on average than proteinencoding genes $(33.13+/-52.66 \mathrm{~kb}$ versus $45.63+/-$ $78.65 \mathrm{~kb}, P<10^{-8}$, Student's's $t$-test). We compared also the size of the 4,496 transcripts corresponding to the 584 lincRNA genes to the size of 97,172 transcripts of the selected protein-encoding genes. The lincRNAs have shorter transcript on average than protein-encoding genes $\left(3.12+/-2.42 \mathrm{~kb}\right.$ versus $3.65+/-2.85 \mathrm{~kb}, P<10^{-45}$, Student's $t$-test). In addition, lincRNA genes show also fewer exons than protein-encoding genes $(3.04+/-2.0$ versus $10.43+/-10.50, P=0$, Student's test).

To determine whether the bovine muscle lincRNAs we detected have the same expression feature, we compared the normalised quantified expression levels of the 584 lincRNAs to that of the 15,358 known selected protein-encoding genes. Our comparison indicates that the identified bovine lincRNAs do show significant lower expression than the proteinencoding genes $\left(4.16 \times 10^{-5}+/-3.70 \times 10^{-4}\right.$ versus $5.62 \times$ $10^{-5},+/-8.44 \times 10^{-4}, P<10^{-3}$, Student's $t$-test). Previous studies also showed that lincRNAs are expressed at significantly lower levels than are protein-coding transcripts $[6,11,40]$. 


\section{LincRNA gene expression}

Not all 584 lincRNA genes were expressed among the nine selected samples. 418 genes were detected in all samples, while 55 genes were detected in less than five samples (Additional file 2: Table S1). The highest number of putative lincRNAs (572 or $97 \%$ of all predicted lincRNAs) was found in sample LIM1.

The sequencing reads derived from the 584 different putative lincRNA made up a bit more than $1 \%$ of all the paired-end reads mapped onto the bovine genome. Three genes encoding putative lincRNAs had each more than $0.05 \%$ of the total mapped paired-end sequencing reads. The gene encoding a putative lincRNA with the most sequencing reads (XLOC_026244) was represented by $0.28 \%$ of the total sequencing reads. These lincRNAs with a relatively high expression level in bovine Longissimus thoraci might play an important role in muscle function.

As our study identified lincRNAs from different samples but from the same tissue, it is possible to analyse the inter-individual variability of the gene expression level of the identified lincRNAs. We calculated the coefficient of variation for each lincRNA, from the expression level measured with the nine different Longissimus thoraci samples and compared it with the gene expression variation of the selected protein-coding genes. Interestingly, there was a significant difference when we compared the expression variation of the 418 lincRNAs with the 10,775 known selected protein-encoding genes found in all nine muscle samples $(46.67+/-30.12$ versus $36.58+/-22.69, P<10^{-11}$, Student's $t$-test). The higher averaged expression variation of the identified lincRNAs suggests a loosened gene expression regulation of these genes compared to protein-encoding genes. To our knowledge, it is the first time that this is described.

The observed higher gene expression variation of lincRNA genes could be due to a higher number of polymorphisms within the regulatory regions. To support this hypothesis we compared the SNP density (number of SNPs per $\mathrm{kb}$ ) of the regulatory region of the lincRNA genes and protein-coding genes. We mapped SNPs from Ensembl (Ensembl Variation version 74) to the upstream $(-10 \mathrm{~kb}$ to $0 \mathrm{bp}$ from the predicted transcription starting site) and downstream (up to $1 \mathrm{~kb}$ after the stop codon) regions of the lincRNA genes and protein-encoding genes. There was no significant difference when we compared the averaged SNP density in the downstream regions of the 418 lincRNAs with the one of the 15,358 known selected protein-encoding genes $\left(7.16 \times 10^{-3}+/-\right.$ $4.66 \times 10^{-3}$ versus $7.09 \times 10^{-3}+/-4.41 \times 10^{-3}, P=0.7 \%$, Student's $t$-test). In addition, there was no significant difference when we compared the averaged SNP density in the upstream regions of the group of genes $\left(7.66 \times 10^{-4}+/-\right.$ $6.34 \times 10^{-4}$ versus $7.20 \times 10^{-4}+/-5.84 \times 10^{-4}, P=0.54 \%$,
Student's $t$-test). The lack of difference in SNP densities do not rule out that the observed higher gene expression variation of lincRNA genes could be due to the effect of polymorphisms within the regulatory regions. However, the SNP densities should be determined after sequencing the whole-genome or the regulatory region of the lincRNA genes and protein-coding genes of the nine animals for which the expression data was generated.

The expression variation of the identified genes might be affected by the moderate sequencing depth obtained for some samples. More work is therefore required to confirm the higher averaged expression variation seen with lincRNA genes compared to protein-coding genes.

\section{Co-expression analysis}

We have in our study the expression levels of the identified lincRNAs and of known protein-encoding genes in nine samples from the same tissue. It is therefore possible to analyse the co-expression of lincRNAs with protein-encoding genes. Using the normalised expression levels, we calculated the Spearman's rank correlation coefficient for each lincRNA with each proteinencoding gene. We found after correction for multiple testing 2,081 pairs of lincRNA/protein-encoding genes showing a highly correlated expression $\left(P<1.11 \times 10^{-8}\right)$ (Additional file 5: Table S3). 45 different lincRNA genes and 966 different protein-encoding genes had their expression highly correlated. 14 lincRNAs showed correlations with more than 20 protein-encoding genes. Four lincRNA genes (XLOC_009350, XLOC_018437, XLOC_021729 and XLOC_024598) had the most correlations, with 192 different protein-encoding genes. All lincRNA/protein-encoding gene pairs showing highly correlated expression had the same correlation and $P$-values $(r h o=1, P<0)$. No anti-correlated lincRNA/ protein-coding gene pairs were found. There were neither no pairs of lincRNA/protein-encoding genes located at less than $2 \mathrm{Mb}$ apart, suggesting a lack of cis-regulation among the paired genes we detected in bovine muscle. However, we found 88,888 co-expression correlations without the Bonferroni correction for multiple testing $(P<=0.05)$ between 95 different lincRNAs and 15,209 different protein-encoding genes, including 1,738 cis and 33,896 anti- correlations.

\section{Validation of novel lincRNAs}

To confirm that the identified bovine lincRNAs are transcribed in vivo, 14 lincRNAs detected in all 9 samples were randomly selected for RT-PCR validation. 13 out of the 14 selected lincRNAs could be amplified using total RNA from Longissimus thoraci, as shown in Figure 1. All amplification products have the expected size; however for lincRNA XLOC_021462 we obtain an extra band of $\sim 1,000 \mathrm{bp}$. The high percentage of validation 
suggests that most putative lincRNAs might be truly expressed in vivo.

\section{Functional lincRNA candidates}

The positions of the 584 genes encoding putative linRNAs were compared to the position on the UMD3.1 bovine genome assembly of know quantitative trait loci (QTLs) deposited in the public database AnimalQTLdb [41]. 556 loci were located in 2,389 different QTL regions, including 507 lincRNAs within 550 QTLs for meat quality/musclerelated traits (Additional file 6: Table S4). For example, 110 different putative lincRNAs are found in 48 QTL regions for meat tenderness; whereas 281 putative lincRNAs are within 86 QTLs for marbling score. QTLs were sorted into two groups (meat quality/muscle-related QTLs versus other QTLs) and the number of lincRNAs found in these two groups were counted. We then performed a Chisquared test and found a significant difference $(P=8.63 \times$ $10^{-63}$ ) in the number of lincRNAs between the two groups (Additional file 7: Table S5), suggesting an enrichment of SNPs in meat/muscle related QTLs. The high number of putative lincRNAs located within known QTL regions, particularly in chromosomal regions harbouring QTLs for meat quality-related traits, indicates that the collection of lincRNAs found in the Longissimus thoraci transcriptome may prove useful to elucidate their effect on mechanisms underlying the genetic variability of meat quality traits.

\section{Conclusions}

The present study represents the first analysis of large intergenic non-coding genes discovered in bovine muscle. Using a computational pipeline that we developed to analyse
RNA-Seq data, we identified 584 different novel putative lincRNAs. We could validate by RT-PCR 13 out of fourteen selected putative lincRNAs, suggesting that most putative lincRNAs might be truly expressed in vivo. The identified putative bovine lincRNA genes share most features with mammalian counterparts.

As our study identified lincRNAs in Longissimus thoraci from nine different Limousin animals, we could analyse the variability of the gene expression level of the identified lincRNAs and compared it with the gene expression variation of the known selected protein-coding genes. We found a higher averaged expression variation for the identified lincRNAs suggesting a loosened gene expression regulation. We also analysed the co-expression of lincRNAs with protein-encoding genes and found 2,093 pairs of lincRNA/protein-encoding genes showing a highly correlated expression.

Some of the lincRNAs expressed in muscle are located within quantitative trait loci for meat quality traits. Future experimental studies are required to unravel the function of these molecules and to elucidate their effect on mechanisms underlying the genetic variability of meat quality traits.

The lincRNAs identified here will complement the catalog of lincRNAs already discovered in cattle and therefore will help to better annotate the bovine genome.

\section{Methods}

\section{Animal ethics}

All animal experimentation complied with the French Veterinary Authorities' rules. No ethics approval was

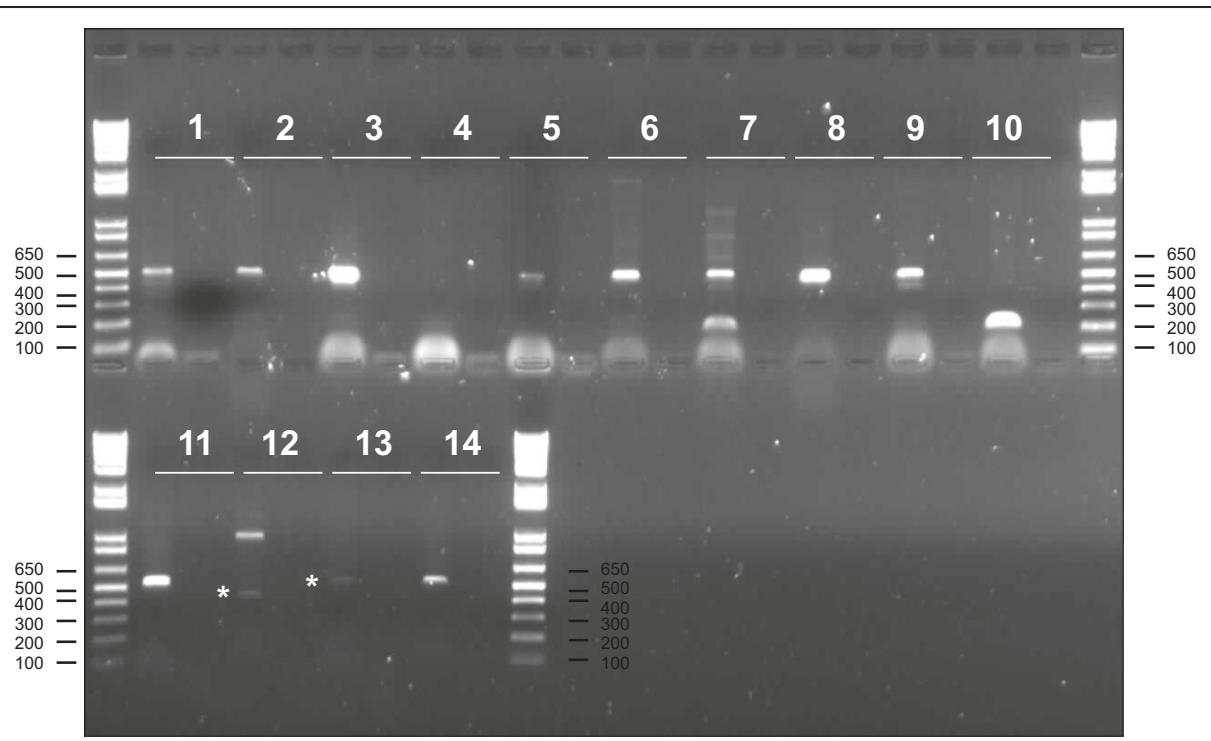

Figure 1 Validation of selected lincRNAs using RT-PCR. Selected lincRNAs are numbered from 1 to 14 as detailed in Additional file 6: Table S4. The first and second lanes are with PCR products using CDNA or without CDNA (negative control), respectively. ${ }^{*}$ indicates faint but specific amplification products. 
required by a specific committee, as the selected animals were not animals bred for experimental reasons.

\section{Animals and tissue samples}

The study was conducted with nine Limousin bull calves from a large study on the genetic determinism of beef and meat quality traits [42]. The nine bull calves were not closely related to one another (for at least 4 generations) were fattened in a single feedlot and fed ad libidum with wet corn silage. They were humanely slaughtered in an accredited commercial slaughterhouse when they reached 16 months. Longissimus thoraci (LT) muscle samples were dissected immediately after death and tissue samples were snap frozen in liquid nitrogen and stored at $-80^{\circ} \mathrm{C}$ until analysis.

\section{RNA isolation and sequencing}

RNA extraction and sequencing were performed as previously described [30]. Briefly, after transfer to ice-cold RNeasy RLT lysis buffer (Qiagen, Courtaboeuf, France), LT tissue samples were homogenised using a Precellys tissue homogeniser (Bertin Technologie, Montigny-leBretonneux, France). Total RNA was isolated using RNeasy Midi columns (Qiagen) and then treated with RNAse-free DNase I (Qiagen) for $15 \mathrm{~min}$ at room temperature according to the manufacturer's protocols. The concentration of total RNA was measured with a Nanodrop ND-100 instrument (Thermo Scientific, Ilkirch, France) and the quality was assessed with an RNA 6000 Nano Labchip kit using an Agilent 2100 Bioanalyzer (Agilent Technologies, Massy, France). All nine samples had an RNA Integrity Number (RIN) value greater than eight.

The mRNA-Seq libraries were prepared using the TruSeq RNA Sample Preparation Kit (Illumina, San Diego, CA) according to the manufacturer's instructions. Briefly, Poly-A containing mRNA molecules were purified from $4 \mu \mathrm{g}$ total RNA of each sample using oligo(dT) magnetic beads and fragmented into 150-400 bp pieces using divalent cations at $94^{\circ} \mathrm{C}$ for $8 \mathrm{~min}$. The cleaved mRNA fragments were converted to double-stranded cDNA using SuperScript II reverse transcriptase (Life Technologies, Saint Aubin, France) and primed by random primers. The resulting cDNA was purified using Agencourt AMPure ${ }^{\circ}$ XP beads (Beckman Coulter, Villepinte, France). Then, cDNA was subjected to end-repair and phosphorylation and subsequent purification was performed using Agencourt AMPure $^{\circ}$ XP beads (Beckman Coulter). These repaired cDNA fragments were 3 '-adenylated producing cDNA fragments with a single ' $\mathrm{A}$ ' base overhung at their 3 '-ends for subsequent adapterligation. Illumina adapters containing indexing tags were ligated to the ends of these 3'-adenylated cDNA fragments followed by two purification steps using Agencourt AMPure ${ }^{\circ}$ XP beads (Beckman Coulter). Ten rounds of PCR amplification were performed to enrich the adapter-modified cDNA library using primers complementary to the ends of the adapters. The PCR products were purified using Agencourt AMPure ${ }^{\circ} \mathrm{XP}$ beads (Beckman Coulter) and size-selected (200 \pm 25 bp) on a $2 \%$ agarose Invitrogen E-Gel (Thermo Scientific). Libraries were then checked on an Agilent Technologies 2100 Bioanalyzer using the Agilent High Sensitivity DNA Kit and quantified by quantitative PCR with the QPCR NGS Library Quantification kit (Agilent Technologies). After quantification, tagged cDNA libraries were pooled in equal ratios and a final qPCR check was performed post-pooling. The pooled libraries were used for $2 \times 100 \mathrm{bp}$ paired-end sequencing on one lane of the Illumina HiSeq2000 with a TruSeq SBS v3-HS Kit (Illumina). After sequencing, the samples were demultiplexed and the indexed adapter sequences were trimmed using the CASAVA v1.8.2 software (Illumina).

\section{Transcriptome assembly and gene expression counts}

RNA-Seq reads from each sample were aligned to the UMD3.1 Bos taurus reference genome [43,44] with TopHat (version 1.4.0) using the default settings and a maximum intron size of 50,000 bp and the expected mean inner distance between paired-reads of $300 \mathrm{bp} \mathrm{(-I}$ $50000-r$ 300) [31]. Only uniquely mapped and properly paired reads were then assembled with Cufflinks [32] (version 2.0) and using Ensembl's bovine gene annotation (version 71). A unique set of all transcripts found among the nine samples was generated using Cuffcompare and all assembled transcripts were quantified in each sample using HTSeq-count ([45], version 0.5.4).

\section{Analysis of coding potential}

Classification of each transcript as either coding or noncoding was determined using a step-wise pipeline.

First, all candidate transcript models were scored with PhyloCSF [46] to determine their coding potential. PhyloCSF uses a multispecies nucleotide sequence alignment to identify conserved protein-coding region, based on a statistical comparison of phylogenetic codon models. We used a five-species alignment between cow, human (hg19), mouse (mm9), rat (rn4) and dog (CanFam2). Pairwise alignments were obtained from the UCSC website (http://hgdownload.soe.ucsc.edu/downloads.html\#cow). All transcripts with a negative score were retained as potential non-coding candidates.

Second, the Coding Potential Assessment Tool (CPAT) [47] was applied (version 1.2.1) on all candidate transcript models in order to assess their coding potential by a second independent prediction method. According to the authors [47] the CPAT coding probability score ranges between 0 and 1 , and the optimum cut-off value for protein coding probability varies depending on the 
species to be analysed. To determine the cut-off value, CPAT was trained using a set of 10,000 bovine known protein-encoding transcripts and a set of 10,000 bovine non-coding sequences. The set of non-coding sequences included 3,801 bovine short non-coding genes and 6,199 bovine intronic sequences (larger than 200 bases). Bovine known protein-encoding transcripts and intronic sequences were extracted randomly from Ensembl using the bovine gene annotation (version 71). The two training sets were randomly split into ten different parts to perform a 10-fold cross-validation analysis. CPAT was trained on one part and the predictions were made on the remaining nine parts. This process was repeated ten times, so each sample was used once for the prediction. Prediction accuracy (sensitivity and specificity) was obtained for each repetition. Sensitivity and specificity were calculated as follows: Sensitivity $=\mathrm{TP} /(\mathrm{TP}+\mathrm{FN})$ and Specificity $=\mathrm{TN} /(\mathrm{TN}+\mathrm{FP})$. Where TP, FP, TN and FN are the numbers of true positives (non-coding sequences predicted to be non-coding), false positives (protein-coding transcripts predicted to be non-coding), true negatives (protein-coding transcripts predicted to be coding) and false negatives (non-coding sequences predicted to be coding). The cut-off value was selected to maximize specificity and sensitivity. In order to extract potential non-coding transcripts with a high reliability from our dataset, all transcripts with a score below 0.348 were retained as potential non-coding RNAs.

Finally, all candidate transcripts were translated in silico into the three possible open reading frames using a custom script and compared against the Pfam protein families database ([48], version 27.0) with the hmmscan algorithm (package HMMER3, version 3.1b1). Candidate transcript models with known protein motifs were discarded.

\section{Validation by RT-PCR}

The RT-PCR primers were designed using Primer3 (http://bioinfo.ut.ee/primer3/) with the optimal PCR product length set between 191 and 513 bp. Primer sequences are presented in Additional file 8: Table S6. The PCR primers were synthesised by Eurofins MWG Operon. Each PCR primer pair was tested using a pool of cDNA made of two animals.

One microgram of DNase I-treated total RNA was used to synthesize the first strand of cDNA using the SuperScript First-Strand Synthesis System III for RTPCR (Invitrogen) according to the manufacturer's instructions and applying a combination of $50 \mathrm{ng}$ random hexamers.

Polymerase chain reactions were performed in $25 \mu \mathrm{l}$ using $60 \mathrm{ng}$ cDNA, $1 \times$ PCR buffer, $1.5 \mathrm{~mm} \mathrm{MgCl}_{2}$, $0.2 \mathrm{~mm}$ of each dNTP, $0.3 \mu \mathrm{M}$ of each primer and $1 \mathrm{U}$ GoTaq DNA polymerase (Promega). The following touchdown cycling protocol was used: $95^{\circ} \mathrm{C}$ for $2 \mathrm{~min}$, followed by 13 cycles of $95^{\circ} \mathrm{C}$ for $1 \mathrm{~min}, 1 \mathrm{~min}$ of annealing (the annealing temperature was progressively lowered from 68 to $56^{\circ} \mathrm{C}$ in steps of $1^{\circ} \mathrm{C}$ every cycle) and $72^{\circ}$ $\mathrm{C}$ for $1 \mathrm{~min}$. These initial cycles were followed by $20 \mathrm{cy}$ cles of $95^{\circ} \mathrm{C}$ for $1 \mathrm{~min}, 55^{\circ} \mathrm{C}$ for $1 \mathrm{~min}$ and $72^{\circ} \mathrm{C}$ for $1 \mathrm{~min}$, and a final extension step at $72^{\circ} \mathrm{C}$ for $2 \mathrm{~min} .10 \mu \mathrm{l}$ of each PCR product was then analysed by gel electrophoresis with a $1 \%$ agarose gel.

\section{Statistical analysis}

Spearman's rank correlation coefficient were calculated for the correlation studies using the statistical $R$ package.

\section{Data availability}

The sequencing data have been submitted to the European Nucleotide Archive (accession numbers ERP002220 and E-MTAB-2646).

\section{Additional files}

\section{Additional file 1: Figure S1. Performance evaluation using 10-fold} cross-validation.

Additional file 2: Table S1. List of candidate lincRNA genes.

Additional file 3: Table S2. List of lincRNA genes previously identified in cattle.

Additional file 4: Figure S2. Distribution of lincRNA genes over all bovine chromosomes.

Additional file 5: Table S3. List of lincRNA/protein-encoding gene pairs with highly correlated expression.

Additional file 6: Table S4. List of putative lincRNAs located within known QTL regions.

Additional file 7: Table S5. Chi-squared test details.

Additional file 8: Table S6. Primer sequences used for RT-PCR.

Competing interests

The authors declare that they have no competing interests.

\section{Authors' contributions}

$\mathrm{CB}$ and $\mathrm{MB}$ carried out the bioinformatics analyses, under the supervision of DG and DR. DE performed the RNA-Seq experiment and ER the RT-PCR work. AD carried out the mapping of the RNA-Seq data, under the supervison of CK. CM prepared the RNA samples. DR conceived the study, analysed the data and drafted the manuscript. All authors read and approved the final manuscript.

\section{Acknowledgements}

We would like to thank the help of the bioinformatics team from the eBio IFB facility (Université Paris-Sud, Orsay). The RNA-Seq work was funded by the INRA Animal Genetics Department (BovRNA-Seq project). The sampling of the Limousin Longissimus thoraci biopsies was part of the Qualvigène project, funded by Agence Nationale de la Recherche (contracts ANR-05-GANI-005 and ANR-05GANI-017-01) and APIS GENE (contract 01-2005-QualviGenA-02). The eBio IFB facility is funded by Agence Nationale de la Recherche (contract ANR-11-INSB-0013). The authors wish to thank the anonymous reviewers for their valuable comments and suggestions, which were helpful in improving our manuscript.

\section{Author details}

${ }^{1}$ INRA, UMR1313, Unité Génétique Animale et Biologie Intégrative, Domaine de Vilvert, F-78352 Jouy-en-Josas, France. ${ }^{2}$ AgroParisTech, UMR1313, Unité Génétique Animale et Biologie Intégrative, Domaine de Vilvert, F-78352 Jouy-en-Josas, France. ${ }^{3}$ Institut de Génétique et Microbiologie, Université Paris-Sud, UMR8621, F-91405 Orsay, France. ${ }^{4}$ CNRS, UMR8621, Institut de 
Génétique et Microbiologie, F-91405 Orsay, France. ${ }^{5}$ INRA, UMR 444 , Laboratoire de Génétique Cellulaire, INRA Auzeville, BP 52627, F-31326 Castanet-Tolosan Cedex, France. ${ }^{6} \mathrm{GeT}-\mathrm{PlaGe}$, Genotoul, INRA Auzeville, BP 52627, F-31362 Castanet-Tolosan Cedex, France. ${ }^{7}$ INRA, SIGENAE, UR 875, INRA Auzeville, BP 52627, F-31326 Castanet-Tolosan Cedex, France.

Received: 13 March 2014 Accepted: 13 June 2014

Published: 19 June 2014

\section{References}

1. Han P, Hang $C T$, Yang J, Chang CP: Chromatin remodeling in cardiovascular development and physiology. Circ Res 2011, 108(3):378-396.

2. Wang KC, Chang HY: Molecular mechanisms of long noncoding RNAs. Mol Cell 2011, 43(6):904-914.

3. Mercer TR, Dinger ME, Sunkin SM, Mehler MF, Mattick JS: Specific expression of long noncoding RNAs in the mouse brain. Proc Natl Acad Sci U S A 2008, 105(2):716-721.

4. Guttman M, Amit I, Garber M, French C, Lin MF, Feldser D, Huarte M, Zuk O, Carey BW, Cassady JP, Cabili MN, Jaenisch R, Mikkelsen TS, Jacks T, Hacohen $\mathrm{N}$, Bernstein BE, Kellis M, Regev A, Rinn JL, Lander ES: Chromatin signature reveals over a thousand highly conserved large non-coding RNAs in mammals. Nature 2009, 458(7235):223-237.

5. Khali AM, Guttman M, Huarte M, Garber M, Raj A, Rivea Morales D, Thomas K, Presser A, Bernstein BE, Van Oudenaarden A, Regev A, Lander ES, Rinn JL: Many human large intergenic noncoding RNAs associate with chromatin-modifying complexes and affect gene expression. Proc Natl Acad Sci U S A 2009, 106(28):11667-11672.

6. Guttman M, Garber M, Levin JZ, Donaghey J, Robinson J, Adiconis X, Fan L, Koziol MJ, Gnirke A, Nusbaum C, Rinn JL, Lander ES, Regev A: Ab initio reconstruction of cell type-specific transcriptomes in mouse reveals the conserved multi-exonic structure of lincRNAs. Nat Biotechnol 2010, 28(5):503-510

7. Brawand D, Soumillon M, Necsulea A, Julien P, Csárdi G, Harrigan P, Weier M, Liechti A, Aximu-Petri A, Kircher M, Albert FW, Zeller U, Khaitovich P, Grützner F, Bergmann S, Nielsen R, Pääbo S, Kaessmann H: The evolution of gene expression levels in mammalian organs. Nature 2011, 478(7369):343-348.

8. Ulitsky I, Shkumatava A, Jan CH, Sive H, Bartel DP: Conserved function of lincRNAs in vertebrate embryonic development despite rapid sequence evolution. Cell 2011, 147(7):1537-1550.

9. Li T, Wang S, Wu R, Zhou X, Zhu D, Zhang Y: Identification of long nonprotein coding RNAs in chicken skeletal muscle using next generation sequencing. Genomics 2012, 99(5):292-298.

10. Nam JW, Bartel DP: Long noncoding RNAs in C. elegans. Genome Res 2012, 22(12):2529-2540.

11. Pauli A, Valen E, Lin MF, Garber M, Vastenhouw NL, Levin JZ, Fan L, Sandelin A, Rinn JL, Regev A, Schier AF: Systematic identification of long noncoding RNAs expressed during zebrafish embryogenesis. Genome Res 2012, 22(3):577-591.

12. Young RS, Marques AC, Tibbit C, Haerty W, Bassett AR, Liu JL, Ponting CP: Identification and properties of 1,119 candidate lincRNA loci in the Drosophila melanogaster genome. Genome Biol Evol 2012, 4(4):427-442.

13. Necsulea A, Soumillon M, Warnefors M, Liechti A, Daish T, Zeller U, Baker JC, Grützner F, Kaessmann H: The evolution of IncRNA repertoires and expression patterns in tetrapods. Nature 2014, 505(7485):635-640.

14. Derrien $T$, Johnson R, Bussotti $G$, Tanzer A, Djebali S, Tilgner H, Guernec $G$, Martin D, Merkel A, Knowles DG, Lagarde J, Veeravalli L, Ruan X, Ruan Y, Lassmann T, Carninci P, Brown JB, Lipovich L, Gonzalez JM, Thomas M, Davis CA, Shiekhattar R, Gingeras TR, Hubbard TJ, Notredame C, Harrow J, Guigó R: The GENCODE v7 catalog of human long noncoding RNAs: Analysis of their gene structure, evolution, and expression. Genome Res 2012, 22(9):1775-1789

15. Bánfai B, Jia H, Khatun J, Wood E, Risk B, Gundling WE Jr, Kundaje A, Gunawardena HP, Yu Y, Xie L, Krajewski K, Strahl BD, Chen X, Bickel P, Giddings MC, Brown JB, Lipovich L: Long noncoding RNAs are rarely translated in two human cell lines. Genome Res 2012, 22(9):1646-1657.

16. ENCODE Project Consortium, Bernstein BE, Birney E, Dunham I, Green ED, Gunter C, Snyder M: An integrated encyclopedia of DNA elements in the human genome. Nature 2012, 489(7414):57-74.

17. Djebali S, Davis CA, Merkel A, Dobin A, Lassmann T, Mortazavi A, Tanzer A, Lagarde J, Lin W, Schlesinger F, Xue C, Marinov GK, Khatun J, Williams BA, Zaleski C, Rozowsky J, Röder M, Kokocinski F, Abdelhamid RF, Alioto T,
Antoshechkin I, Baer MT, Bar NS, Batut P, Bell K, Bell I, Chakrabortty S, Chen $X$, Chrast J, Curado J, et al: Landscape of transcription in human cells. Nature 2012, 489(7414):101-108.

18. Kapranov P, Cheng J, Dike S, Nix DA, Duttagupta R, Willingham AT, Stadler PF, Hertel J, Hackermüller J, Hofacker IL, Bell I, Cheung E, Drenkow J, Dumais E, Patel S, Helt G, Ganesh M, Ghosh S, Piccolboni A, Sementchenko V, Tammana H, Gingeras TR: RNA maps reveal new RNA classes and a possible function for pervasive transcription. Science 2007, 316(5830):1484-1488

19. Babak T, Blencowe BJ, Hughes TR: A systematic search for new mammalian noncoding RNAs indicates little conserved intergenic transcription. BMC Genomics 2005, 6:104.

20. Bono H, Yagi K, Kasukawa T, Nikaido I, Tominaga N, Miki R, Mizuno Y, Tomaru Y, Goto H, Nitanda H, Shimizu D, Makino H, Morita T, Fujiyama J, Sakai T, Shimoji T, Hume DA, Hayashizaki Y, Okazaki Y, RIKEN GER Group; GSL Members: Systematic expression profiling of the mouse transcriptome using RIKEN CDNA microarrays. Genome Res 2003, 13(6B):1318-1323.

21. Gibb EA, Brown CJ, Lam WL: The functional role of long non-coding RNA in human carcinomas. Mol Cancer 2011, 10:38

22. Ramsköld D, Wang ET, Burge CB, Sandberg R: An abundance of ubiquitously expressed genes revealed by tissue transcriptome sequence data. PLoS Comput Biol 2009, 5(12):e1000598.

23. Mercer TR, Dinger ME, Mattick JS: Long non-coding RNAs: insights into functions. Nat Rev Genet 2009, 10(3):155-159.

24. Ponting $\mathrm{CP}$, Oliver $\mathrm{PL}$, Reik W: Evolution and functions of long noncoding RNAs. Cell 2009, 136(4):629-641.

25. Wang $X$, Song $X$, Glass CK, Rosenfeld MG: The long arm of long noncoding RNAs: roles as sensors regulating gene transcriptional programs. Cold Spring Harb Perspect Biol 2011, 3:a003756.

26. Wutz A, Gribnau J: X inactivation Xplained. Curr Opin Genet Dev 2007, $17: 387-393$

27. Qu Z, Adelson DL: Bovine ncRNAs are abundant, primarily intergenic, conserved and associated with regulatory genes. PLOS ONE 2010, 7(8):e42638.

28. Huang W, Long N, Khatib H: Genome-wide identification and initial characterization of bovine long non-coding RNAs from EST data. Anim Genet 2012, 43(6):674-682.

29. Weikard R, Hadlich F, Kuehn C: Identification of novel transcripts and noncoding RNAs in bovine skin by deep next generation sequencing. BMC Genomics 2013, 14:789.

30. Djari A, Esquerré D, Weiss B, Martins F, Meersseman C, Boussaha M, Klopp C, Rocha D: Gene-based single nucleotide polymorphism discovery in bovine muscle using next-generation transcriptomic sequencing. BMC Genomics 2013, 14:307.

31. Trapnell C, Pachter L, Salzberg SL: TopHat: discovering splice junctions with RNA-Seq. Bioinformatics 2009, 25(9):1105-1111.

32. Trapnell C, Williams BA, Pertea G, Mortazavi A, Kwan G, Van Baren MJ, Salzberg SL, Wold BJ, Pachter L: Transcript assembly and quantification by RNA-Seq reveals unannotated transcripts and isoform switching during cell differentiation. Nat Biotechnol 2010, 28(5):511-515.

33. Canovas A, Rincon G, Islas-Trejo A, Wickramasinghe S, Medrano JF: SNP discovery in the bovine milk transcriptome using RNA-Seq technology. Mamm Genome 2010, 21:592-598.

34. Huang W, Nadeem A, Zhang B, Babar M, Soller M, Khatib H: Characterization and comparison of the leukocyte transcriptomes of three cattle breeds. PLoS ONE 2012, 7:e30244.

35. Wickramasinghe S, Rincon G, Islas-Trejo A, Medrano JF: Transcriptional profiling of bovine milk using RNA sequencing. BMC Genomics 2012, 13:45.

36. Baldwin RL 6th, Wu S, Li W, Li C, Bequette BJ, Li RW: Quantification of transcriptome responses of the rumen epithelium to butyrate infusion using RNA-seq technology. Gene Regul Syst Bio 2012, 6:67-80.

37. Li RW, Rinaldi M, Capuco AV: Characterization of the abomasal transcriptome for mechanisms of resistance to gastrointestinal nematodes in cattle. Vet Res 2011, 42:114.

38. Driver AM, Peñagaricano F, Huang W, Ahmad KR, Hackbart KS, Wiltbank MC: RNA-Seq analysis uncovers transcriptomic variations between morphologically similar in vivo- and in vitro-derived bovine blastocysts. BMC Genomics 2012, 13:118.

39. Xie C, Yuan J, Li H, Li M, Zhao G, Bu D, Zhu W, Wu W, Chen R, Zhao Y: NONCODEv4: exploring the world of long non-coding RNA genes. Nucleic Acids Res 2014, 42:D98-D103. 
40. Cabili MN, Trapnell C, Goff L, Koziol M, Tazon-Vega B, Regev A, Rinn JL: Integrative annotation of human large intergenic noncoding RNAs reveals global properties and specific subclasses. Genes Dev 2011, 25(18):1915-1927.

41. Hu ZL, Fritz ER, Reecy JM: AnimalQTLdb: a livestock QTL database tool set for positional QTL information mining and beyond. Nucleic Acids Res 2007, 35:D604-D609.

42. Allais S, Levéziel H, Payet-Duprat N, Hocquette JF, Lepetit J, Rousset S: The two mutations Q204X and nt821 of the myostatin gene affect carcass and meat quality in heterozygous young bulls of French beef breeds. J Anim Sci 2010, 88:446-454.

43. Zimin AV, Delcher AL, Florea L, Kelley DR, Schatz MC, Puiu D, Hanrahan F, Pertea G, Van Tassell CP, Sonstegard TS, Marçais G, Roberts M, Subramanian P, Yorke JA, Salzberg SL: A whole-genome assembly of the domestic cow, Bos taurus. Genome Biol 2009, 10:R42.

44. Bovine Genome S, Analysis C, Elsik CG, Tellam RL, Worley KC, Gibbs RA, Muzny DM, Weinstock GM, Adelson DL, Eichler EE, Elnitski L, Guigó R, Hamernik DL, Kappes SM, Lewin HA, Lynn DJ, Nicholas FW, Reymond A, Rijnkels M, Skow LC, Zdobnov EM, Schook L, Womack J, Alioto T, Antonarakis SE, Astashyn A, Chapple CE, Chen HC, Chrast J, Câmara F, et al: The genome sequence of taurine cattle: a window to ruminant biology and evolution. Science 2009, 324(5926):522-528.

45. HTSeq. http://www-huber.embl.de/users/anders/HTSeq/doc/overview.html.

46. Lin MF, Jungreis I, Kellis M: PhyloCSF: a comparative genomics method to distinguish protein coding and non-coding regions. Bioinformatics 2011, 27(13):i275-i282.

47. Wang L, Park HJ, Dasari S, Wang S, Kocher JP, Li W: CPAT: Coding-Potential Assessment Tool using an alignment-free logistic regression model. Nucleic Acids Res 2013, 41(6):e74.

48. Sonnhammer EL, Eddy SR, Durbin R: Pfam: a comprehensive database of protein domain families based on seed alignments. Proteins 1997, 28(3):405-420.

doi:10.1186/1471-2164-15-499

Cite this article as: Billerey et al:: Identification of large intergenic non-coding RNAs in bovine muscle using next-generation transcriptomic sequencing. BMC Genomics 2014 15:499.

\section{Submit your next manuscript to BioMed Central and take full advantage of:}

- Convenient online submission

- Thorough peer review

- No space constraints or color figure charges

- Immediate publication on acceptance

- Inclusion in PubMed, CAS, Scopus and Google Scholar

- Research which is freely available for redistribution 